\title{
The SAAAVE Act and Routine Ambulatory Medical Care Fail to Diagnose Patients with Abdominal Aortic Aneurysms prior to Rupture: A Single-Institution Experience
}

\author{
Kamell Eckroth-Bernard, Robert P. Garvin, Evan J. Ryer, \\ James R. Elmore, and David P. Franklin \\ Department of Vascular and Endovascular Surgery, Geisinger Medical Center, Danville, PA 17821, USA \\ Correspondence should be addressed to Robert P. Garvin; rpgarvin@geisinger.edu
}

Received 10 December 2012; Accepted 8 January 2013

Academic Editors: P. Schoenhagen, S. Takebayashi, and M. Vasse

Copyright (c) 2013 Kamell Eckroth-Bernard et al. This is an open access article distributed under the Creative Commons Attribution License, which permits unrestricted use, distribution, and reproduction in any medium, provided the original work is properly cited.

\begin{abstract}
Objectives. To demonstrate that routine ambulatory medical evaluation in the outpatient setting and current utilization of the Screening Abdominal Aortic Aneurysms Very Efficiently (SAAAVE) Act are inadequate methods to diagnose abdominal aortic aneurysms (AAA) prior to rupture. Methods. A retrospective review was performed on patients with ruptured AAA (rAAA) at a single institution. All patients who were evaluated in an ambulatory care setting within 6 months of the date of rupture were identified, and clinical data were analyzed. Results. Between January 1, 2004 and December 31, 2010, there were 149 patients with rAAA. Fifty-two of 149 (34.9\%) patients were evaluated in the outpatient setting within 6 months prior to the date of rupture, and these patients form the basis of this study. Thirty-six of 52 (70\%) patients were male, average age was 73.5 years, average BMI was 28 , and average aneurysm diameter was $76 \mathrm{~mm}$. Only 5/52 (9.6\%) patients had physical exam findings suspicious for AAA. Only 9/52 (17\%) would have been eligible for the screening abdominal ultrasound under the SAAAVE Act. Conclusions. Routine medical evaluation in the ambulatory care setting and current utilization of AAA screening methods are inadequate at detecting AAA in the at-risk population prior to rupture.
\end{abstract}

\section{Introduction}

Ruptured abdominal aortic aneurysm (rAAA) is the 15th leading cause of death in the United States accounting for more than 8500 hospital deaths per year [1]. Despite greater public awareness and advances in the delivery of vascular care, the overall mortality for rAAA still ranges from $80 \%$ to $90 \%$ [2-5]. In contrast, elective repair of abdominal aortic aneurysms (AAA) carries a mortality of $<5 \%$ at most high volume centers [6-8]. With this in mind, it is intuitive that the most effective method of reducing AAA related mortality is the early identification and elective repair of AAAs prior to rupture, assuming patients are of acceptable surgical risk [9].

In an effort to reduce the number of AAA-related deaths, the United States Congress approved the Screening Abdominal Aortic Aneurysms Very Efficiently (SAAAVE) Act in January 2007. The SAAAVE Act permits a single screening ultrasound examination as part of the "Welcome to Medicare" package for patients with defined risk factors for AAA. Specifically, males between the age of 65 and 75 years who have smoked greater than 100 cigarettes or patients of any age/gender with a strong family history are eligible for this screening examination [10].

Despite the introduction of the SAAAVE Act, screening for AAA remains underutilized with less than 10,000 beneficiaries being screened in 2007 [11]. More recent data suggests that even after several years of implementation, the SAAAVE Act has not had any discernible effect on AAA rupture rate or death rate, presumably due to underutilization [12]. Reasons for underutilization are likely multifactorial and include a general lack of familiarity with the SAAAVE Act, lack of appropriate history taking to identify high risk patients, and the general belief that AAAs can be easily palpated on physical examination by health care providers. In addition, rigid requirements must be met for primary care physicians to be fully reimbursed for this screening 
ultrasound; thus, the amount of physician work level and subsequent decreased reimbursement may also be contributing factors to SAAAVE Act underutilization [12]. A recent systematic review evaluating AAA screening guidelines reached a consensus supporting the use of a one-time screening of elderly men to detect and treat AAAs $\geq 5.5 \mathrm{~cm} \mathrm{[13].} \mathrm{However,}$ the same study found no screening consensus for other target groups (men $\leq 65$ years, women, etc.), stressing the need for further study to better define screening criteria, effective management of small AAAs, improved prediction models, and cost-effectiveness analyses. The aim of this study was to investigate our hypothesis that routine medical evaluation in the ambulatory care setting and current utilization of the SAAAVE Act in its current form, are not effective methods of screening and diagnosing patients at risk for AAA.

\section{Methods}

This study was approved by the Geisinger Medical Center Institutional Review Board. All patients presenting to Geisinger Medical Center with a diagnosis of rAAA between January 1, 2004 and December 31, 2010 were identified by Current Procedural Terminology (CPT) and International Classification of Diseases 9th revision (ICD-9) codes. These selected patients were then retrospectively reviewed through the use of the electronic health record (EHR) and a clinical decision intelligence system (CDIS) data warehouse to verify the correct diagnosis. The CDIS data warehouse at Geisinger Medical Center receives updated data every 24 hours from the EHR as well as regular data updates from multiple other source systems, including hospital billing, financial decision support, insurance claims, and high-use third-party reference datasets. Any patient with a rAAA who was evaluated in an outpatient setting within 6 months of the date of rupture was included in the study.

Demographics, cardiovascular risk factors, physical exam findings, prior radiologic evaluations, and preadmission medications were obtained from the EHR and/or CDIS. In addition, radiographic imaging with abdominal ultrasound (Duplex) and computed tomography (CT) scan prior to aneurysm rupture was independently reviewed separately by a vascular surgeon and a radiologist for each patient to establish whether the diagnosis of AAA was previously established or overlooked. Aneurysm diameter (the greatest transverse measurement perpendicular to the center line) at the time of rupture was measured by direct review of the radiographic images or, if not available, it was taken from the operating surgeon's documentation within the EHR. Data were analyzed using Society for Vascular Surgery reporting standards [14]. The Fisher exact test was used for analysis of categorical variables. Subset analyses were performed using the unpaired $t$-test for continuous variables. A $P$ value of less than .05 was used to determine statistical significance.

\section{Results}

3.1. Clinical Characteristics. Between January 1, 2004 and December 31, 2010, 149 patients presented to Geisinger
TABLE 1: Demographics, medical comorbidities, and current medical therapy in 52 patients evaluated in the outpatient setting $\leq 6$ months of diagnosis of ruptured AAA.

\begin{tabular}{|c|c|c|}
\hline Variable & Mean \pm SD & $\begin{array}{c}\text { Total } 52 \\
n(\%)\end{array}$ \\
\hline Age (years) & $73.5 \pm 8.9$ & \\
\hline Male & & $36(69)$ \\
\hline Female & & $16(31)$ \\
\hline Hypertension & & $42(80.8)$ \\
\hline Peripheral vascular disease & & $13(25)$ \\
\hline Diabetes & & $15(28.8)$ \\
\hline Hyperlipidemia & & $32(61.5)$ \\
\hline Coronary artery disease & & $27(51.9)$ \\
\hline Chronic obstructive pulmonary disease & & $20(38.5)$ \\
\hline Congestive heart failure & & $5(9.6)$ \\
\hline Chronic kidney disease & & $19(36.5)$ \\
\hline \multicolumn{3}{|l|}{ Smoking } \\
\hline Never & & $4(7.7)$ \\
\hline Current & & $21(40.4)$ \\
\hline Quit & & $16(30.8)$ \\
\hline Unknown & & $11(21.1)$ \\
\hline Aspirin & & $30(55.6)$ \\
\hline Clopidogrel & & $5(9.3)$ \\
\hline Beta blocker & & $28(51.9)$ \\
\hline Statin & & $27(50)$ \\
\hline ACEI/ARB & & $24(44.4)$ \\
\hline
\end{tabular}

AAA: abdominal aortic aneurysm; ACEI: angiotensin converting enzyme inhibitor; ARB: angiotensin receptor blocker; chronic kidney disease, GFR $<60$.

Medical Center with a diagnosis of rAAA. Demographics and cardiovascular risk factors are detailed in Table 1 demonstrating a typical distribution of associated medical comorbidities in a population of patients with abdominal aortic aneurysm disease.

\subsection{Ambulatory Care Evaluation and Physical Examination} Comparisons. Of the 149 patients presenting with a rAAA, 52 patients $(34.9 \%)$ had an EHR documented outpatient evaluation in a Geisinger Health System ambulatory care setting within 6 months of the date of rupture, and these 52 patients form the basis of this study (Figure 1). These 52 patients had complete outpatient and inpatient EHR information, whereas the other $97 / 149$ patients either lacked complete outpatient EHR information due to having primary care visits outside of the Geisinger system or had evaluations in the Geisinger system greater than 6 months prior to the date of rupture and were therefore excluded from the analysis. The 52 study patients had been evaluated by several different types of medical specialists within 6 months (mean 52.4 days) prior to the date of rupture, most of these specialists being either primary care physicians or cardiologists (Table 2). Of note, 3 of these patients had been seen in the vascular surgery clinic within 6 months of the date of rupture and were deemed poor candidates for elective surgical repair. 


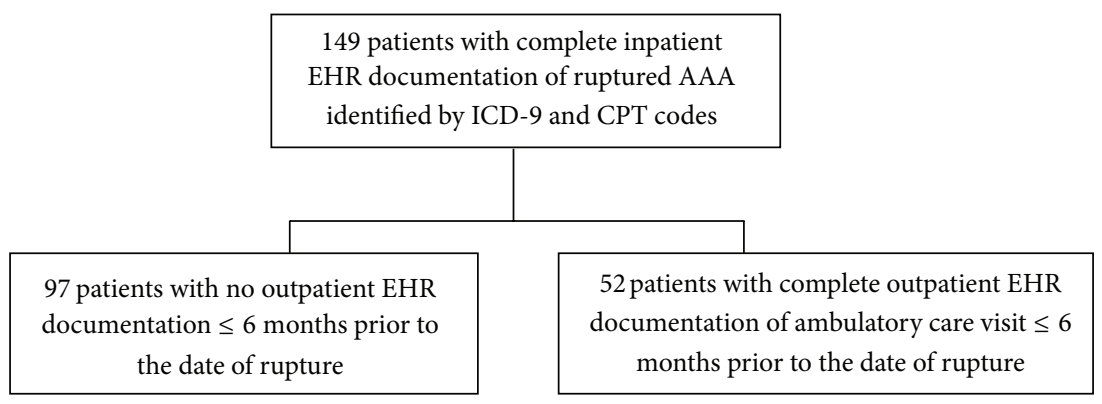

FIGURE 1: Reference diagram of 149 patients diagnosed with ruptured abdominal aortic aneurysm and availability of both inpatient and outpatient complete EHR data. CPT, Current Procedural Terminology; ICD-9, International Classification of Diseases ninth revision; EHR, electronic health record.

TABLE 2: Medical specialty and average time (days) from ambulatory care visit for 52 patients evaluated $\leq 6$ months prior to diagnosis of ruptured AAA.

\begin{tabular}{lcc}
\hline Variable & Days (range) & $\begin{array}{c}\text { Total } \\
n(\%)\end{array}$ \\
\hline Outpatient visit_rAAA & $52.4(0-166)$ & \\
PCP & & $(57.7)$ \\
Cardiology & $7(13.5)$ \\
Ophthalmology & $4(7.7)$ \\
Vascular Surgery & $3(5.8)$ \\
Dermatology & $2(3.8)$ \\
Hematology/Oncology & $1(1.9)$ \\
Urology & $1(1.9)$ \\
Infectious disease & $1(1.9)$ \\
Nephrology & $1(1.9)$ \\
Cardiothoracic & $1(1.9)$ \\
Orthopedics & $1(1.9)$ \\
\hline
\end{tabular}

PCP: primary care provider; AAA: abdominal aortic aneurysm.

Outpatient abdominal examinations were documented in $36 / 52$ (69.2\%) patients revealing 2 patients with abdominal tenderness alone, 1 patient with a pulsatile mass alone, and 1 patient with both abdominal tenderness and pulsatile mass (Table 3 ). The one patient with both tenderness and pulsatile mass was appropriately referred to the emergency department for evaluation and indeed was diagnosed with a rAAA and survived open repair. In comparison, study group abdominal exam findings by the vascular fellow upon patient arrival to the emergency department for rAAA revealed 12/52 (23.1\%) patients with tenderness alone $(P=.008), 10 / 52$ $(19 \%)$ patients with pulsatile mass alone $(P=.004)$, and $16 / 52(30.8 \%)$ patients with both tenderness and pulsatile mass $(P<.0001)$. Thus, a palpable pulsatile abdominal mass with or without tenderness was only present in $26 / 52$ (50\%) of the patients evaluated by a vascular surgeon in the emergent inpatient setting at the time of rupture.

The 52 study group patients had a mean BMI of $28.2 \mathrm{~kg} / \mathrm{m}^{2}$ and a mean AAA diameter of $76 \mathrm{~mm}$. BMI and AAA size were evaluated in patients with and without palpable pulsatile masses in the emergency department (Table 4).
TABle 3: Comparison of outpatient physical exams findings in 38 patients versus vascular surgery fellow physical examination findings in 52 patients.

\begin{tabular}{lccc}
\hline Variable & ACP total 38 & VSF total 52 & \multirow{2}{*}{$\begin{array}{c}\text { Pvalue } \\
\end{array}$} \\
& $2(\%)$ & $n(\%)$ & \\
\hline Tenderness & $1(1.9)$ & $12(23.1)$ & .008 \\
Pulsatile mass & $10(19)$ & .004 \\
Tenderness + pulsatile mass & $1(1.9)$ & $16(30.8)$ & $<.0001$ \\
\hline Total exam & $4(7.7)$ & $38(73.1)$ & $<.00001$ \\
\hline
\end{tabular}

ACP: ambulatory care provider in outpatient setting; VSF: vascular surgery fellow in emergency department setting.

TABLe 4: BMI, AAA size, and vascular fellow examination for pulsatile abdominal mass of the 52 study patient presenting with ruptured AAA.

\begin{tabular}{lcc}
\hline Variable & Mean \pm SD & Range \\
\hline BMI $\left(\mathrm{kg} / \mathrm{m}^{2}\right)$ without pulsatile mass & $28.9 \pm 6.0$ & $16.1-52.0$ \\
BMI $\left(\mathrm{kg} / \mathrm{m}^{2}\right)$ with pulsatile mass & $27.7 \pm 7.1$ & $16.1-52.0$ \\
AAA size $(\mathrm{mm})$ without pulsatile mass & $73.2 \pm 18.4$ & $44.0-120$ \\
AAA size $(\mathrm{mm})$ with pulsatile mass & $79.5 \pm 18.4$ & $55.0-120$ \\
\hline
\end{tabular}

BMI: body mass index; AAA: abdominal aortic aneurysm.

When patients with palpable pulsatile masses were compared with patients without palpable pulsatile masses, there was no significant difference in either the BMI $(P=.53)$ or AAA size $(P=.22)$.

3.3. Radiologic Findings and Referral. Twenty-one (40.3\%) patients underwent 27 radiographic studies (Duplex and/or CT scan) of the abdomen and/or pelvis prior to the date of rupture. Thirteen of these patients had radiographic evidence of an AAA within 1 year of presenting with an rAAA and 8 patients had radiographic evidence of an AAA more than a year prior to rupture. Three patients had Duplex alone, 12 patients had CT scan alone, and 6 patients underwent both imaging modalities. Twelve of these 21 patients were referred and evaluated in the vascular surgery clinic based on the results of these imaging studies. Of these 12 patients, 4 declined repair, 4 were followed for AAA sizes $<50 \mathrm{~mm}, 2$ patients were offered repair and ruptured in the interim $(2$ 
TABLE 5: SAAAVE Act eligibility in 52 study patients with ruptured $\mathrm{AAA}$ at the time of presentation.

\begin{tabular}{lcccc}
\hline Variable & $\begin{array}{c}\text { Total (52) } \\
n(\%)\end{array}$ & $\begin{array}{c}\text { Smoking } \\
n\end{array}$ & $\begin{array}{c}\text { FHx } \\
n\end{array}$ & $\begin{array}{c}\text { Eligible } \\
n(\%)\end{array}$ \\
\hline Age (male) & & & & \\
$65-75$ & $10(19.2)$ & 7 & 0 & $7(13.5)$ \\
$<65$ & $7(13.5)$ & 5 & 0 & 0 \\
$>75$ & $19(36.5)$ & 11 & 1 & $1(1.9)$ \\
Female & $16(30.8)$ & 13 & 1 & $1(1.9)$ \\
\hline Total & & & & $(17.3)$ \\
\hline
\end{tabular}

AAA: abdominal aortic aneurysm; Smoking: smoking history (>100 cigarettes); FHx: family history.

weeks and 3 weeks) after evaluation, and 2 patients (one with AAA diameter of $40 \mathrm{~mm}$ and the other with AAA diameter of $67 \mathrm{~mm}$ at the time of diagnosis) were lost to followup. The remaining 9 patients were not evaluated in the vascular surgery clinic prior to the date of rupture. Reasons for the lack of referral could not be determined based on review of the EHR.

3.4. SAAAVE Act Eligibility. Per the current SAAAVE Act criteria, only $9 / 52(17.3 \%)$ study patients would have been eligible for the Welcome to Medicare screening aortic ultrasound (Table 5 ). Two (3.8\%) of the 52 patients ( 1 female and 1 male) had a documented family history of AAA in the EHR. The single female patient underwent an abdominal ultrasound for abdominal pain demonstrating an incidental $3.1 \mathrm{~cm}$ AAA. The patient was referred and followed in the vascular surgery clinic for 5 years. Upon routine vascular followup, she was admitted for acute expansion, found to have a contained rAAA, and underwent a successful open repair. The single male patient ( $>75$ years of age) had a family history of AAA but did not have any prior radiographic screening. The remaining 7 male patients did not have documented radiographic AAA screening studies. Of the male study patients, 7/36 (13.5\%) patients were less than 65 years old, and 19/36 (36.5\%) were greater than 75 years old. Only 10/36 (19.2\%) male patients were between the age of 65 and 75 years, seven of which were eligible for the screening ultrasound secondary to tobacco use. Eleven of the 19 male patients greater than 75 years old had a history of smoking. Twentythree $(63.8 \%)$ of the male patients smoked or had a history of smoking. There were 16/52 (30.8\%) females between the age of 61 and 96 years. Thirteen $(81.3 \%)$ of the females had a smoking history.

\section{Discussion}

The aim of this study was to investigate whether current screening guidelines, in conjunction with routine ambulatory medical care evaluation, are an effective way to identify as well as screen patients at risk for AAA. Our review identified that only $17 \%$ of patients who presented to our institution with an rAAA would have been eligible for a screening ultrasound based upon the SAAAVE Act criteria at the time of rupture. These findings suggest that current AAA screening guidelines are not adequate to make a substantial reduction in aneurysm related mortality. Given the high mortality associated with rAAA, expanding screening criteria may have enabled earlier detection in the remaining $83 \%$ of our patients, thus providing the opportunity for referral and elective repair.

Our findings, while being of great concern, also fail to account for those who qualify for an aneurysm screening but do not utilize it. As others have documented, achieving a high initial rate of screening attendance, along with a low elective operative mortality rate, is vital for any meaningful decrease in AAA-related mortality [15]. It is not entirely clear why screening for other disease processes, such as breast, cervical, and colon cancer, is utilized to a much greater degree than AAA screening. It is likely that increased education and greater familiarity with the aforementioned disease processes account for differing utilization rates. These differences highlight the importance of continued efforts to educate the public and our primary care colleagues regarding AAAs. Over time, we hope that increased awareness, acceptance, and adherence will lead to a reduction in aneurysm related mortality.

Moreover, we also need to educate primary care providers about the specialty of vascular surgery and the services we provide, for patient referral to a vascular surgeon is an important step in the elective AAA repair process. It is a curious finding that $40 \%$ of our study patients had radiographic evidence of AAA prior to rupture, but many were not referred and evaluated in the vascular surgery clinic. Similar findings were reported by MacDonald et al. who retrospectively identified a known AAA in 34/104 (33\%) patients presenting with rAAA [16]. Of these patients studied by MacDonald, 16 were deemed unfit for elective surgery, 6 were lost to followup, and 12 were awaiting elective AAA repair.

Another interesting finding of our investigation, albeit less novel, is that physical examination (PE) is not adequate to diagnose an AAA. In the outpatient ambulatory medical setting, despite most of these 52 patients have been seen and evaluated by primary care physicians and cardiologists within 6 months of rupture, the diagnosis of AAA based on physical exam was rare. These findings were somewhat anticipated and are not meant to be an indictment against our medical colleagues, but rather emphasize the fact that in routine real world experience, patients at risk for AAA often go undiagnosed when simple history and physical exam are employed in the outpatient care setting. Even in the experienced hands of vascular surgery fellows concerned about the diagnosis of AAA, physical examination was only accurate about $50 \%$ of the time on the actual day of rupture. Perhaps altered hemodynamics and abdominal distention played a role in masking the physical exam findings by the vascular fellows in the emergency department, but the results nonetheless further emphasize that physical exam alone is not reliable in diagnosing rAAA even in this population of patients with large aneurysms and modest BMI values. Other authors have reported similar findings. Specifically, Anjum et al. described the difficulty of aortic palpation in obese patients prior to elective open AAA repair in 1995 [17]. In 
their study, 38\% of patients had AAAs initially detected by PE and the remaining $62 \%$ were found incidentally on radiologic examinations. Patients with AAAs detected by PE had lower BMIs $\left(23.7 \pm 3.6 \mathrm{~kg} / \mathrm{m}^{2}\right)$ regardless of the AAA size. Our data suggest that neither AAA size nor BMI exerts a strong influence on whether or not an AAA is palpable as a pulsatile mass (mean BMI $27.7 \pm 7.1 \mathrm{~kg} / \mathrm{m}^{2}$ and mean AAA size of $76 \mathrm{~mm}$ ), so we, therefore, advocate radiographic screening as mandatory regardless of $\mathrm{PE}$ findings in high-risk patients.

Lastly, a significant gender issue exists regarding AAA screening for women resulting in missed opportunities for earlier detection. Approximately $30 \%$ of our study patients were women, but only one of these women (based on family history) would have been SAAAVE Act eligible. Multiple articles have documented the lower prevalence of AAA in women versus men. In 2007, DeRubertis et al. came to a similar conclusion documenting AAA prevalence rates of $0.7 \%$ and $3.9 \%$ for women and men, respectively [18]. However, the DeRubertis study discovered that the prevalence of AAA significantly increases to $6.4 \%$ in women with multiple atherosclerotic risk factors (increasing age, cardiovascular disease, and history of tobacco use). In our opinion, excluding women with AAA risk factors is unwarranted.

There are several limitations to our study. The total number of study group patients is small due to our exclusion of patients not evaluated within 6 months of the date of rupture. It is also quite likely that many of the remaining 97/149 patients were in fact seen in the ambulatory setting within 6 months of rupture but were outside of our health system and therefore not eligible for the study due to a lack of complete outpatient EHR data. In regard to the accuracy of the outpatient visit physical examination findings, to be fair to the primary care physicians and cardiologists, many of the patients were likely being evaluated for other medical problems and underwent focused exams of other organ systems, thus accounting for the fact that only $36 / 52$ patients had outpatient abdominal examinations documented in the EHR. Also, the physical examinations performed in the emergent department by the vascular fellows were biased due to the vascular fellows' awareness of the likely diagnosis of rAAA. Lastly, this is a retrospective study without any comparison group or potential for randomization.

\section{Conclusion}

Routine medical evaluation in the primary care setting fails to diagnose patients at risk for ruptured abdominal aortic aneurysm. Improved utilization of the one-time screening ultrasound exam covered by Medicare in the SAAAVE Act and continued education of our primary care colleagues are needed in order to increase the diagnostic yield for detecting AAA in high risk patients.

\section{Acknowledgments}

The authors would like to thank Jove Graham and his research staff from the Henry Hood Center for Health Research at Geisinger Medical Center for efficiently facilitating the data collection process for this project.

\section{References}

[1] Prevention CfDCa, "Leading causes of death, United States, 2000-2005," 2008, http://www.cdc.gov/.

[2] H. Bengtsson and D. Bergqvist, "Ruptured abdominal aortic aneurysm: a Population-Based Study," Journal of Vascular Surgery, vol. 18, no. 1, pp. 74-80, 1993.

[3] D. J. Adam, I. V. Mohan, W. P. Stuart, M. Bain, and A. W. Bradbury, "Community and hospital outcome from ruptured abdominal aortic aneurysm within the catchment area of a regional vascular surgical service," Journal of Vascular Surgery, vol. 30, no. 5, pp. 922-928, 1999.

[4] M. Heikkinen, J. P. Salenius, and O. Auvinen, "Ruptured abdominal aortic aneurysm in a well-defined geographic area," Journal of Vascular Surgery, vol. 36, no. 2, pp. 291-296, 2002.

[5] I. Kantonen, M. Lepäntalo, M. Brommels, M. Luther, J. P. Salenius, and K. Ylönen, "Mortality in ruptured abdominal aortic aneurysms," European Journal of Vascular and Endovascular Surgery, vol. 17, no. 3, pp. 208-212, 1999.

[6] R. F. Gillum, "Epidemiology of aortic aneurysm in the United States," Journal of Clinical Epidemiology, vol. 48, no. 11, pp. 12891298, 1995.

[7] R. M. Greenhalgh, L. C. Brown, G. P. Kwong, J. T. Powell, and S. G. Thompson, "EVAR trial participants. Comparison of endovascular aneurysm repair with open repair in patients with abdominal aortic aneurysm (EVAR trial 1), 30 day operative mortality results: randomized controlled trial," The Lancet, vol. 364, pp. 843-848, 2004.

[8] The United Kingdom EVAR Trial Investigators, "Endovascular versus open repair of abdominal aortic aneurysm," The New England Journal of Medicine, vol. 362, no. 20, pp. 1863-1871, 2010.

[9] A. R. Pasch, J. J. Ricotta, and A. G. May, "Abdominal aortic aneurysm: the case for elective resection," Circulation, vol. 70, no. 3, pp. I1-I4, 1984.

[10] E. S. Lee, E. Pickett, N. Hedayati, D. L. Dawson, and W. C. Pevec, "Implementation of an aortic screening program in clinical practice: implications for the Screen for Abdominal Aortic Aneurysms Very Efficiently (SAAAVE) Act," Journal of Vascular Surgery, vol. 49, no. 5, pp. 1107-1111, 2009.

[11] Society for Vascular Surgery, "SAAAVE Act Background," 2010, http://vascularweb.org.

[12] J. B. Shreibati, L. C. Baker, M. A. Hlatky, and M. W. Mell, "Impact of the Screening Abdominal Aortic Aneurysms Very Efficiently (SAAAVE) Act on abdominal ultrasonography use among medicare beneficiaries," Archives of Internal Medicine, vol. 172, no. 19, pp. 1456-1462, 2012.

[13] B. S. Ferket, N. Grootenboer, E. B. Colkesen et al., "Systematic review of guidelines on abdominal aortic aneurysm screening," Journal of Vascular Surgery, vol. 55, no. 5, pp. 1296.e4-1304.e4, 2012.

[14] E. L. Chaikof, M. F. Fillinger, J. S. Matsumura et al., "Identifying and grading factors that modify the outcome of endovascular aortic aneurysm repair," Journal of Vascular Surgery, vol. 35, no. 5, pp. 1061-1066, 2002.

[15] A. Chervu, G. P. Clagett, R. J. Valentine, S. I. Myers, and P. J. Rossi, "Role of physical examination in detection of abdominal aortic aneurysms," Surgery, vol. 117, no. 4, pp. 454-457, 1995.

[16] A. J. MacDonald, O. Faleh, G. Welch, and S. Kettlewell, "Missed opportunities for the detection of abdominal aortic aneurysms," European Journal of Vascular and Endovascular Surgery, vol. 35, no. 6, pp. 698-700, 2008. 
[17] Anjum, R. von Allmen, R. Greenhalgh, and J. T. Powell, "Explaining the decrease in mortality from abdominal aortic aneurysm rupture," British Journal of Surgery, vol. 99, pp. 637645, 2012.

[18] B. G. DeRubertis, S. M. Trocciola, E. J. Ryer et al., "Abdominal aortic aneurysm in women: prevalence, risk factors, and implications for screening," Journal of Vascular Surgery, vol. 46, no. 4, pp. 630.el-635.e1, 2007. 


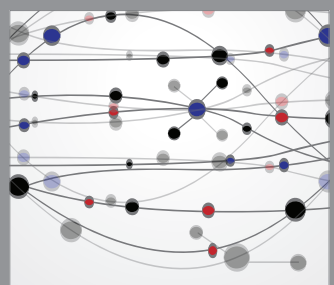

The Scientific World Journal
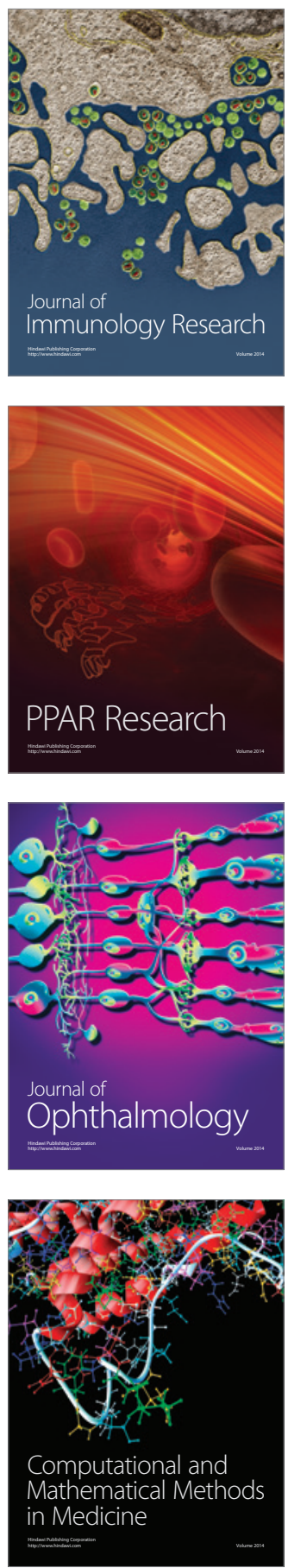

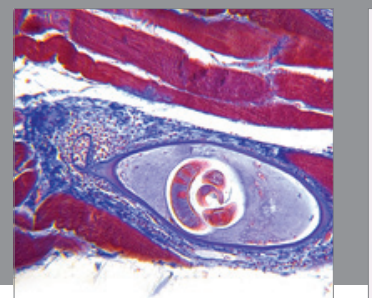

Gastroenterology

Research and Practice
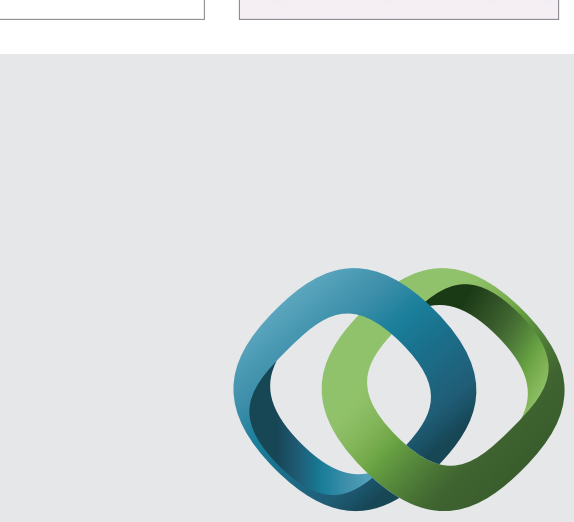

\section{Hindawi}

Submit your manuscripts at

http://www.hindawi.com
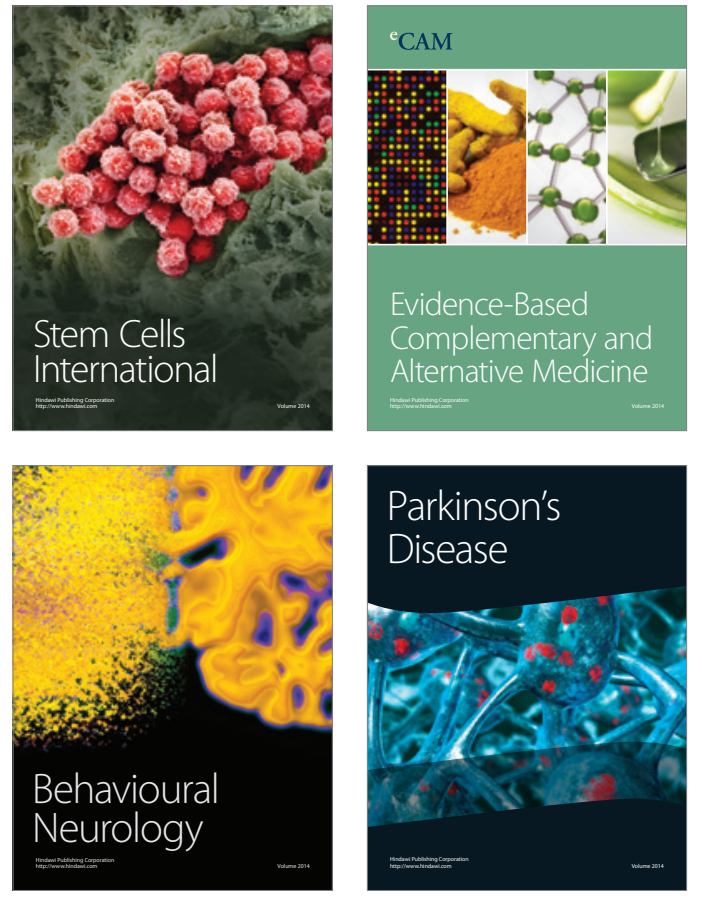
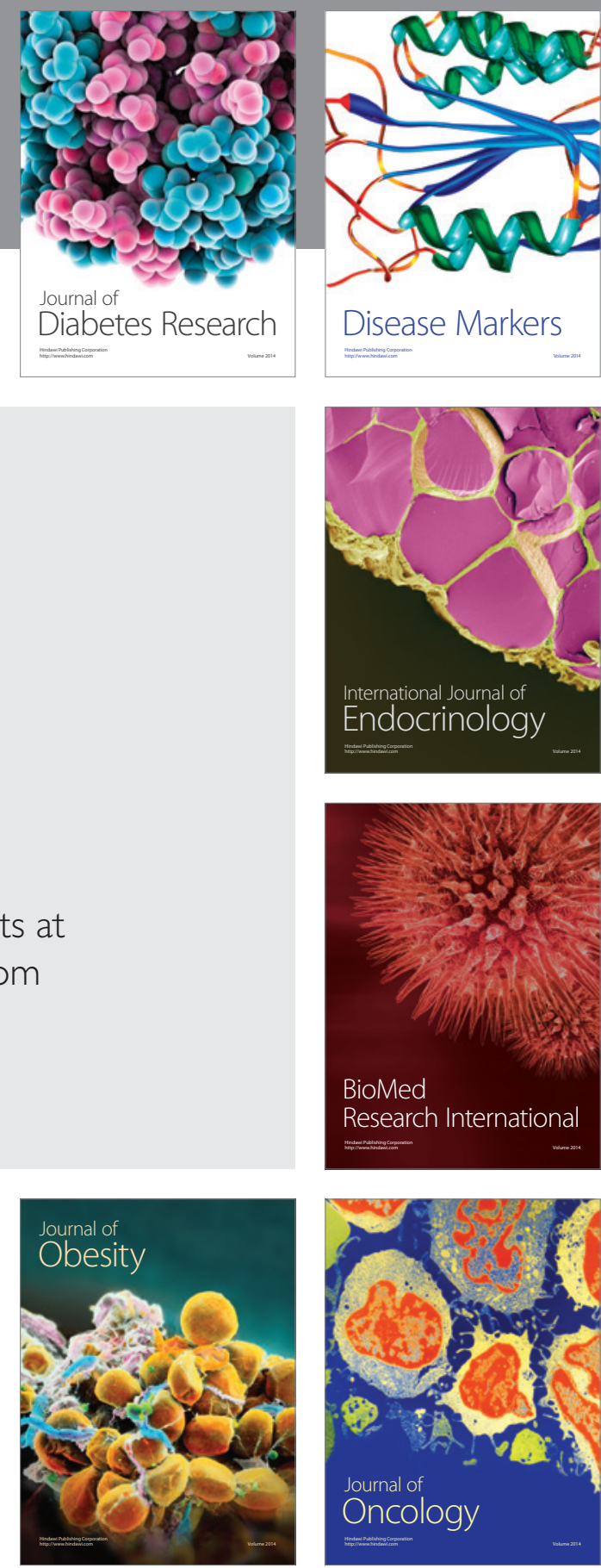

Disease Markers
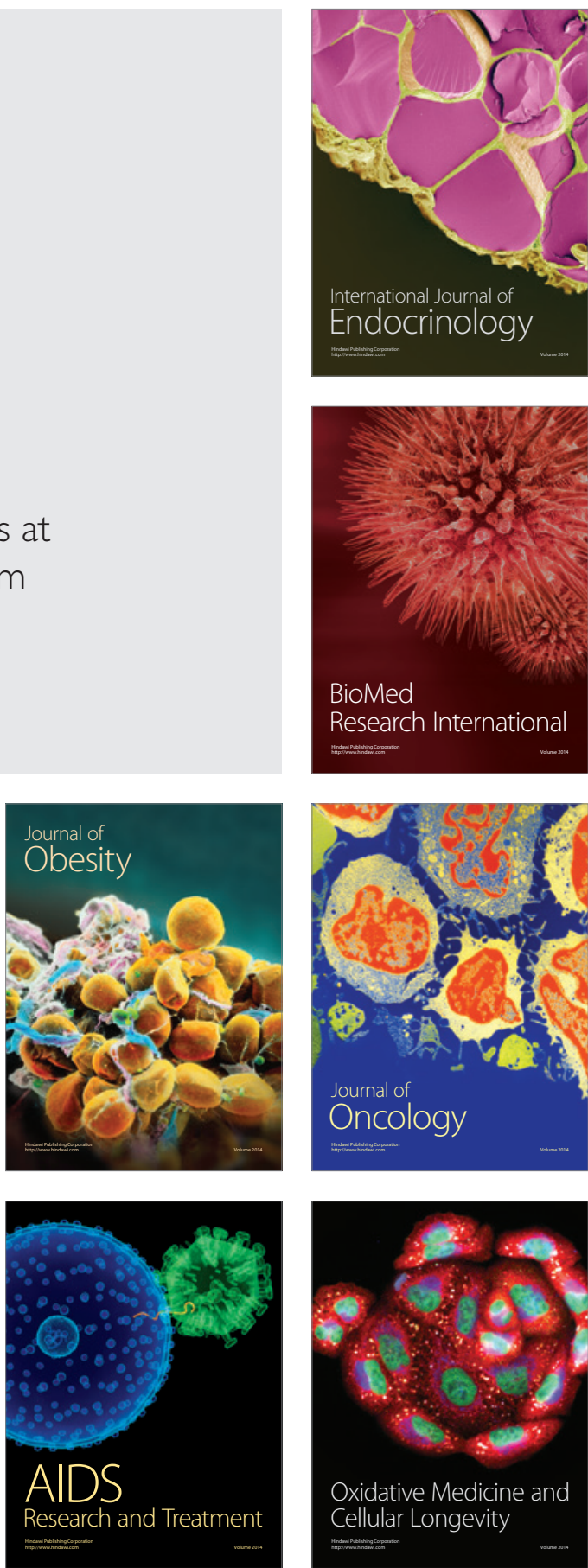